\title{
Effect of Gradual Mobilization with Bed Activity on Hemodynamic Parameters in Patients Undergoing Sleeve Gastrectomy
}

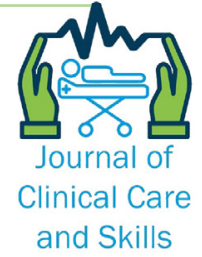

\section{ARTICLE INFO}

\section{Article Type}

Original Research

\section{Authors}

Koohpeyma M. ${ }^{1} M S c$,

Sadat S.J.*2 $M S c$,

Zoladl M. ${ }^{3} P h D$,

Afrasiabifar A. ${ }^{2} P h D$

How to cite this article
Koohpeyma M, Sadat S.J, Zoladl M,
Afrasiabifar A. Effect of Gradual Mo-
bilization with Bed Activity on He-
modynamic Parameters in Patients
Undergoing Sleeve Gastrectomy. Jo-
urnal of Clinical Care and Skills.
2020;1(3):139-146.

\section{A B S T RA C T}

Aims Among different treatments for obesity, sleeve gastrectomy has been more effective. Despite the positive effect of this surgery on the treatment of obesity, sleeve gastrectomy, like other invasive interventions, results in an imbalance in hemodynamic parameters. The aim of this study was to investigate the effect of gradual mobilization with bed activity on hemodynamic parameters in patients undergoing sleeve gastrectomy.

Materials \& Methods In this randomized clinical trial, 88 patients candidate for sleeve gastrectomy in Ghadir Mother and Child Hospital affiliated to the Shiraz University of Medical Sciences, who were eligible, were selected by convenience sampling method and divided into the intervention and control groups (44 subjects in each group) using random block allocation. Hemodynamic parameters, including systolic blood pressure, diastolic blood pressure, and arterial oxygen saturation of all patients were recorded at the time of admission to the ward immediately, 6, 12 and $24 \mathrm{~h}$ after withdrawal from the bed. Data were analyzed by SPSS 21 software and descriptive and inferential statistics.

Findings The changes of hemodynamic parameters in the intervention group were significantly different at each time point of measurement after the interventions compared to these changes in control group $(\mathrm{p}<0.05)$.

Conclusion The effect of gradual mobilization with bed activity is more than the conventional procedure for patients' mobilization after gastrectomy on balancing of systolic blood pressure, diastolic blood pressure, and arterial oxygen saturation.

Keywords Gradual Mobilization; Motion; Hemodynamic; Gastrectomy
"Laparoscopic Research Center" and "Surgery Department, Medicine Faculty", Shiraz University of Medical Sciences, Shiraz, Iran

${ }^{2}$ Nursing Department, Nursing \& Midwifery Faculty, Yasuj University of Medical Sciences, Yasuj, Iran

${ }^{3}$ Social Determinants of Health Research Center, Yasuj University of Medical Sciences, Yasuj, Iran

\section{*Correspondence}

Address: Nursing Department, Nursing \& Midwifery Faculty, Yasuj University of Medical Sciences, Yasuj, Iran. Postal Code: 7591947901

Phone: +98 (74) 33234115

Fax: +98 (74) 33234115

sadatsaiedjavad@gmail.com

\section{Article History}

Received: December 18,2018

Accepted:July 04, 2019

ePublished: September 21, 2020

\section{CITATION L I N KS}

[1] Current status of laparoscopic ... [2] The effect of central obesity on the auditory ... [3] Costeffectiveness of bariatric surgery: should it... [4] Prevalence of obesity in subjects aged 50 ... [5] Correlation between nutritional behavior, obesity ... [6] The psychology of eating: from healthy to ... [7] Screening and interventions for obesity in ... [8] Update on obesity ... [9] Laparoscopic mini-gastric bypass: short ... [10] Effective factors of mini gastric bypass surgery ... [11] Hospital complication rates with bariatric ... [12] Bariatric surgery in patients with morbid ... [13] Use of complementary and alternative medicine ... [14] The second international consensus summit ... [15] Weight loss, appetite suppression, and changes ... [16] Medium-term follow-up results with ... [17] Medium-term follow-up results with ... [18] The effect of bariatric surgery on ... [19] Severe orthostatic hypotension following ... [20] New-onset orthostatic intolerance following ... [21] Orthostatic intolerance following laparoscopic ... [22] The neuropathic postural tachycardia ... [23] Evaluation and treatment of orthostatic ... [24] Postbariatric surgery deaths, which fall under ... [25] Effect of early mobilization on hemodynamic ... [26] The impact of extended bed rest on the ... [27] Clinical practice guidelines for the perioperative ... [28] The quantity of early upright mobilisation ... [29] Effect of passive movements of lower ... [30] Postoperative nursing care of gastric ... [31] Physiologic changes during ... [32] Effectiveness of passive range of motion ... [33] Blood flow velocity and vascular resistance... [34] "Passive exercise" using whole body periodic ... [35] The effects of therapeutic passive movement on ... [36] Effects of mobilization on hemodynamic and ... [37] Respiratory and hemodynamic responses ... 


\section{Introduction}

Obesity and overweight are a serious public health challenge worldwide [1]. Obesity is the accumulation of excess body fat that can have detrimental effects on health [2]. Obesity is regulated by factors, such as metabolism, appetite, diet and physical activity. Although these factors are influenced by genetic characteristics, they are often associated with environmental changes [3]. The prevalence of obesity in adulthood is between $10 \%$ and $40 \%$ worldwide. In Iran, the prevalence of overweight and obesity in adults has been reported to be $30 \%$ and $40 \%$, respectively [4]. The World Health Organization estimates that by 2030, the number of overweight people will reach 1.35 billion and over 573 million adults will become obese [5].

Weight loss in patients with morbid obesity can improve quality of life, mental health and social functioning [6]. Medical treatments for patients with severe obesity include reduced calorie intake, increased physical activity, changing eating habits, and the use of drugs and foods that reduce weight [7]. Many therapeutic approaches with different efficacy have been proposed to address this multidimensional and pervasive phenomenon [8]. According to the National Institutes of Health consensus, bariatric surgery has been more successful than the various methods of treating obesity for patients with Body Mass Index (BMI) of above 35 [9].

Bariatric surgery has other benefits besides weight loss, including improving cardiovascular disease and a $35 \%$ reduction in mortality [10]. In the last two decades, bariatric surgery has been the second abdominal surgery in terms of prevalence [11]. Bariatric surgery involves several surgical procedures performed in obese patients. According to the National Institute of Health guidelines, patients with BMI of equal to or greater than 40 $\mathrm{kg} / \mathrm{m}^{2}$ or equal to or greater than 35 and has other life-threatening conditions, such as diabetes type 2, hypertension and cardiovascular disease, they are candidates for bariatric surgery. Of the three common bariatric surgeries, two methods of laparoscopic adjustable gastric banding (LAGB) and laparoscopic Roux-en-Y gastric bypass (LRYGB) are more common [12]. Laparoscopic sleeve gastrectomy (LSG) is a newer method that has been used as the most common treatment for obesity since 2014 [13]. In this procedure, by removing the large stomach bent, the volume of the stomach reaches 100-150 cc and the remainder forms a tube. In addition, serum ghrelin (appetite regulator) decreases due to the removal of its producing cells [14, 15]. Bariatric surgeries are on the rise in the world due to the long-term effects of weight loss. In addition, it has been very effective in treating obesity disorders [1618]. Following the bariatric surgery, the prevalence of autonomic system dysfunction has increased, characterized by orthostatic intolerance with an unknown cause. However, its cause is weight loss following surgery $[19,20]$. Orthostatic intolerance refers to a group of heterogeneous hemodynamic regulatory abnormalities characterized by inadequate brain perfusion after changing from sleeping to sitting or standing positions. Syncope, syncope-related fatigue, palpitations, exercise intolerance, cognitive impairment, headache, and fatigue are symptoms of orthostatic intolerance [21]. Different forms of orthostatic intolerance include postural orthostatic tachycardia and postural hypotension that occur after bariatric surgery. Postural tachycardia can be defined as an increase in heart rate while standing without orthostatic hypotension [22]. Orthostatic hypotension is referred to as hypotension in standing posture with tachycardia [21].

Orthostatic hypotension is a decrease in systolic blood pressure (20 $\mathrm{mmHg}$ ) or a decrease in diastolic blood pressure $(10 \mathrm{mmHg})$ within 3 minutes following standing position relative to blood pressure levels in sitting or lying down [23].

In a retrospective study by Billakanty et al., the incidence of orthostatic intolerance was evaluated in 15 patients with a mean weight of 124.22 pounds undergoing bariatric surgery. They examined the autonomic response of patients using a gradient table test and found that $53 \%$ had neurocardiogenic reactions, 20\% had autonomic dysfunction, and 20\% had postural tachycardia [20].

The complications of this surgery are divided into long- and short-term complications. Short-term complications include imbalance in hemodynamic parameters, including hypoventilation and decreased arterial oxygen saturation (SPO2) and blood pressure imbalance [15, 24]. The usual care after surgery is resting in bed until vital signs persist and the patient regains consciousness, whereas absolute and prolonged rest does not have a scientific basis and is mostly based on experience and taste [25]. Inactivity and prolonged resting may cause heart failure, which affects the central and peripheral cardiovascular system. The stroke volume during the first month of rest is reduced by $30 \%$, which is associated with an increase in resting heart rate. Symptoms of orthostatic intolerance develop within the first 72 hours of inactivity. The progression of atelectasis and the increased likelihood of respiratory complications are also negative effects of immobility on the respiratory system [26]. Accordingly, patients undergoing long surgeries or patients with high body mass and who have undergone sleeve gastrectomy are encouraged for mobilization after surgery [27]. In several relevant studies, after abdominal surgery, patients are encouraged to a gradual mobilization in to minimize surgical effects on hemodynamic parameters [28]. One of the simplest and cheapest interventions to 
combat these complications is physical activity that increases blood flow and improves heart function, enhances patients' quality of life, and reduces hospital stay ${ }^{[29] .}$

Given that unique nursing considerations and the use of critical thinking skills are essential to meet the needs of patients after bariatric surgery, the challenges of the healthcare team are understandings of bariatric surgery and its complications and they should plan carefully to care for these patients to achieve optimal results. As many patients choose bariatric gastric surgery to treat morbid obesity, skill in intensive care, advanced care, and general nursing care will have a profound effect on the outcome of these patients [30]. Therefore, it is necessary to use routine care that accelerates post-operative hemodynamic indices, and also since no study has been conducted in this field, the purpose of this study was to evaluate the effect of gradual mobilization with bed activity on hemodynamic parameters in patients undergoing sleeve gastrectomy to achieve better care.

\section{Materials and Methods}

This study is a randomized clinical trial, which was performed on volunteer patients of sleeve gastrectomy in Ghadir Mother and Child Hospital affiliated to the Shiraz University of Medical Sciences in 2016. Sample size was calculated 88 individuals ( $n=44$ per group) based on an alpha of $0.01(99 \%$ confidence level), beta of $20 \%$ ( $80 \%$ test power) and $10 \%$ probability of reduction. According to the fourblock random allocation process, a number was assigned to each of the 24 possible arrangements that by matching 22 random numbers with these numbers, the method of arranging 88 samples and assigning each number from one to 88 to the intervention (gradual mobilization) and the control groups and random block allocation list were developed. Then 88 patients undergoing sleeve gastrectomy referred to Ghadir Mother and Child Hospital affiliated to the Shiraz University of Medical Sciences who were eligible based on inclusion criteria, such as willingness to participate in the study, the age of 18-55 years, reading and writing literacy, body mass index of $30 \mathrm{Kg} / \mathrm{m}^{2}$ and above, no history of respiratory disease, no history of mental disorders and no history of smoking and drugs were selected through available sampling method and after obtaining informed consent, based on randomized block allocation list, were assigned into the intervention and control groups. According to the inclusion criteria, none of the intervention and control groups had musculoskeletal pain due to postoperative conditions, abnormalities during surgery, such as vascular damage and extra gastric bypass, intolerance to the intervention protocol, and gastric leak after surgery.

In this study, all aspects of research ethic were observed, for example, obtaining informed consent from legal authorities of the samples, making them informed about the possibility of withdrawal from the study at any time, confidentiality of data, no changes in medications used, and deprivation of the subjects from standard treatments and care. After selecting the subjects, demographic and background characteristics including age, sex, body mass index and their hemodynamic parameters, such as systolic blood pressure, diastolic blood pressure, and mean arterial pressure were measured and recorded using the S740 Bladder Monitor (Saadat; Iran).

The necessary trainings were given by to two research assistants, a male and a female nurse with more than five years of working experience in teachback method. They provided research interventions using an executive protocol for patients with a same gender. In the control group, patients were encouraged to walk on a routine basis, at least once on the first day, and three times on the second day, depending on the patient's mood and willingness, regardless of the time. During hospitalization and at the considered time, the complications of surgery, including arterial oxygen saturation and blood pressure, were assessed.

In the intervention group, after sleeve gastrectomy and gaining full consciousness, gradual mobilization with activity in bed was performed during the following procedure: After entering the ward, the patient was initially placed on back. During the first two hours, the patient's bed was raised about 15 degrees. Then, at the third and fourth hours, the bed head height increased to 30 degrees and 45 degrees, respectively. At the fifth and sixth hours, as the bed head was returned to 15 degrees, the patients were encouraged to move to the left and right. After successful movement, they were asked to change their upper and lower extremities in the normal range of motion. At the seventh hour, the patients were seated for 5 minutes. They were then allowed to sit on the bedside chair for about 10 to 15 minutes. Eventually, after this step, if the patient had no problem, he or she would walk around the corridor assisted by patient's companion, after which the patients would walk as the routine.

While walking, one person cared the patient all the time to prevent the patient from falling. After this stage, during admission and at specified times, i.e. at the entering the ward (first time), after the first mobilization (second time), 6 hours after mobilization (third time), 12 hours after mobilization (fourth time) and 24 hours after mobilization (fifth time), hemodynamic parameters, including arterial oxygen saturation, systolic blood pressure and diastolic blood pressure were evaluated.

Data collection began in late June 2016 and continued until the sample size was completed in late September of that year. In order to prevent bias, the researcher at the baseline was blinded to the intervention and did not know about assigning to 
the intervention or control groups. Through the research, he was also blinded to the intervention and did not know about assigning to the intervention or control groups and recorded hemodynamic parameters, including systolic blood pressure, diastolic blood pressure, and arterial oxygen saturation of all patients at the time of arrival to the ward and immediately, 6, 12 and 24 hours after mobilization. In addition to the researcher, the statistical specialist was blinded to the intervention and control groups until the end of the analysis.

Data were analyzed by SPSS 21 software using descriptive statistics and inferential statistics tests according to the normal distribution of study variables (Parametric tests, including chi-square, independent t-test and analysis of variance with repeated measures for normal distributed data and nonparametric tests, such as Mann-Whitney, Kruskal-Wallis and Friedman tests for data with no normal distribution).

\section{Findings}

Hospitalized patients included 24 men and 64 women with a mean age $34.6 \pm 8.2$ years and BMI $44.4 \pm 4.8 \mathrm{~kg} / \mathrm{m}^{2}$. They underwent sleeve gastrectomy for $155.1 \pm 34.9 \mathrm{~min}$ and were transferred to the surgical ward after $89.8 \pm 35.4 \mathrm{~min}$. Patients in the intervention and control groups were withdrawn from bed after $417.6 \pm 39.1$ and $361.0 \pm 78.0 \mathrm{~min}$, respectively.

At baseline, there was no significant difference between the intervention and control groups in terms of qualitative demographic variables, including sex, marital status, education level and occupation ( $p>0.05$; Table 1$)$.

Also, the intervention and control groups were homogenized in terms of quantitative demographic variables, including mean age, body mass index, duration of operation, length of stay in recovery room, and arterial oxygen saturation percentage after surgery and were not significantly different ( $p>0.05)$. However, dependent variables, such as systolic and diastolic blood pressure had a statistically significant difference at baseline $(\mathrm{p}<0.05)$. Accordingly, these two dependent variables were considered as covariates in the statistical analysis, and the effect of this inconsistency eliminated in the reported results of intergroup comparisons using repeated measures analysis of variance.

There was a significant difference in systolic blood pressure between the intervention and control groups $(\mathrm{p}<0.05)$. Also, changes in systolic blood pressure in the intervention group were significantly different from those in the control group ( $\mathrm{p}<0.05$; Table 2).

Diastolic blood pressure was significantly decreased in the intervention group compared to the control group $(\mathrm{p}<0.05)$. In the intra-group comparison, diastolic blood pressure showed a statistically significant difference $(\mathrm{p}<0.05)$, although a decrease in diastolic blood pressure was significant at different measurement times in the intervention group $(\mathrm{p}<0.05)$, but in the control group, there was no statistically significant difference between its value at different measurement times ( $p>0.05$; Table 3 ).

Inter-group comparison showed that the increase in arterial oxygen saturation immediately after mobilization and $6 \mathrm{~h}$ after mobilization was similar in the intervention and control groups ( $p>0.05)$. However, at 12 and $24 \mathrm{~h}$ after the initial mobilization, these differences were statistically significant between the intervention and control groups $(\mathrm{p}<0.05)$. Intera-group comparison showed a statistically significant difference between the two groups in different measurement times of arterial oxygen saturation $(\mathrm{p}<0.05$; Table 3$)$.

The rate of systolic and diastolic blood pressure decrease and their descending slope were significantly higher in the intervention group than the control group. Also, the increase and the ascending slope of arterial oxygen saturation were significantly higher in the intervention group than the control group (Diagram 1 to 3 ).

Table 1) Comparison of absolute and relative frequency of qualitative demographic variables of patients undergoing sleeve gastrectomy in the intervention and control groups (44 patients per group) at the baseline using chi-square test (numbers in parentheses are percentage)

\begin{tabular}{lccc}
\hline \multicolumn{1}{c}{ Qualitative variables } & Intervention group & Control group & Total \\
\hline Gender & $13(29.5)$ & $11(25.0)$ & $24(27.3)$ \\
Male & $31(70.5)$ & $33(75.0)$ & $64(72.7)$ \\
Female & & & \\
Marital status & $23(52.3)$ & $15(34.1)$ & $38(43.2)$ \\
Single & $21(47.7)$ & $29(65.9)$ & $50(56.8)$ \\
Married & & & 0.63 \\
Education level & $3(6.8)$ & $5(11.4)$ & $8(9.1)$ \\
Below diploma & $15(34.1)$ & $15(34.1)$ & $30(34.1)$ \\
Diploma & $26(59.1)$ & $24(54.5)$ & $50(56.8)$ \\
Above diploma & & & \\
Occupation & $8(18.2)$ & $3(6.8)$ & $11(12.5)$ \\
Unemployed & $4(9.1)$ & $5(11.4)$ & $9(10.2)$ \\
Non-governmental & $8(18.2)$ & $14(31.8)$ & $22(25.0)$ \\
Employed & $16(36.3)$ & $18(40.9)$ & $34(38.7)$ \\
Housewife & $8(18.2)$ & $4(9.1)$ & $12(13.6)$ \\
Student & & & 0.16 \\
\hline
\end{tabular}


Table 2) Comparison of demographic variables and quantitative dependent variables of patients undergoing sleeve gastrectomy in the intervention and control groups at the baseline

\begin{tabular}{|c|c|c|c|}
\hline Quantitative variables & Mean & Statistic & P. value \\
\hline \multicolumn{4}{|l|}{ Age (year) } \\
\hline Intervention group & $32.9 \pm 7.1$ & 1.95 & $* 0.06$ \\
\hline Control group & $36.3 \pm 8.9$ & 1.95 & \\
\hline \multicolumn{4}{|l|}{ BMI $\left(\mathrm{kg} / \mathrm{m}^{2}\right)$} \\
\hline Intervention group & $43.5 \pm 3.1$ & 1.8 & $* 0.08$ \\
\hline Control group & $45.3 \pm 5.9$ & & 0.00 \\
\hline \multicolumn{4}{|l|}{ Duration of surgery (min) } \\
\hline Intervention group & $150.3 \pm 38.9$ & & $* * 0.18$ \\
\hline Control group & $159.9 \pm 30.0$ & -1.3 & 0.18 \\
\hline \multicolumn{4}{|l|}{ Length of stay in recovery room (min) } \\
\hline $\begin{array}{l}\text { Intervention group } \\
\text { Control group }\end{array}$ & $\begin{array}{l}86.8 \pm 34.8 \\
92.7 \pm 36.2\end{array}$ & -1.1 & ${ }^{* *} 0.26$ \\
\hline \multicolumn{4}{|l|}{ Systolic Blood Pressure (mmHg) } \\
\hline $\begin{array}{l}\text { Intervention group } \\
\text { Control group }\end{array}$ & $\begin{array}{c}151.4 \pm 9.7 \\
144.2 \pm 11.4\end{array}$ & -3.2 & ${ }^{*} 0.002$ \\
\hline \multicolumn{4}{|l|}{ Diastolic Blood Pressure (mmHg) } \\
\hline $\begin{array}{l}\text { Intervention group } \\
\text { Control group }\end{array}$ & $\begin{array}{c}93.3 \pm 7.0 \\
84.9 \pm 11.9\end{array}$ & -4.1 & ${ }^{*} 0.0001$ \\
\hline \multicolumn{4}{|l|}{ Arterial oxygen saturation percentage } \\
\hline $\begin{array}{l}\text { Intervention group } \\
\text { Control group }\end{array}$ & $\begin{array}{l}89.0 \pm 2.0 \\
88.2 \pm 1.8\end{array}$ & -2.2 & ${ }^{* *} 0.06$ \\
\hline
\end{tabular}

*Independent t-test; ** Mann-Whitney test

Table 3) Comparison of intra-group and inter-group hemodynamic parameters of patients undergoing sleeve gastrectomy in the study groups according to the time of measurement

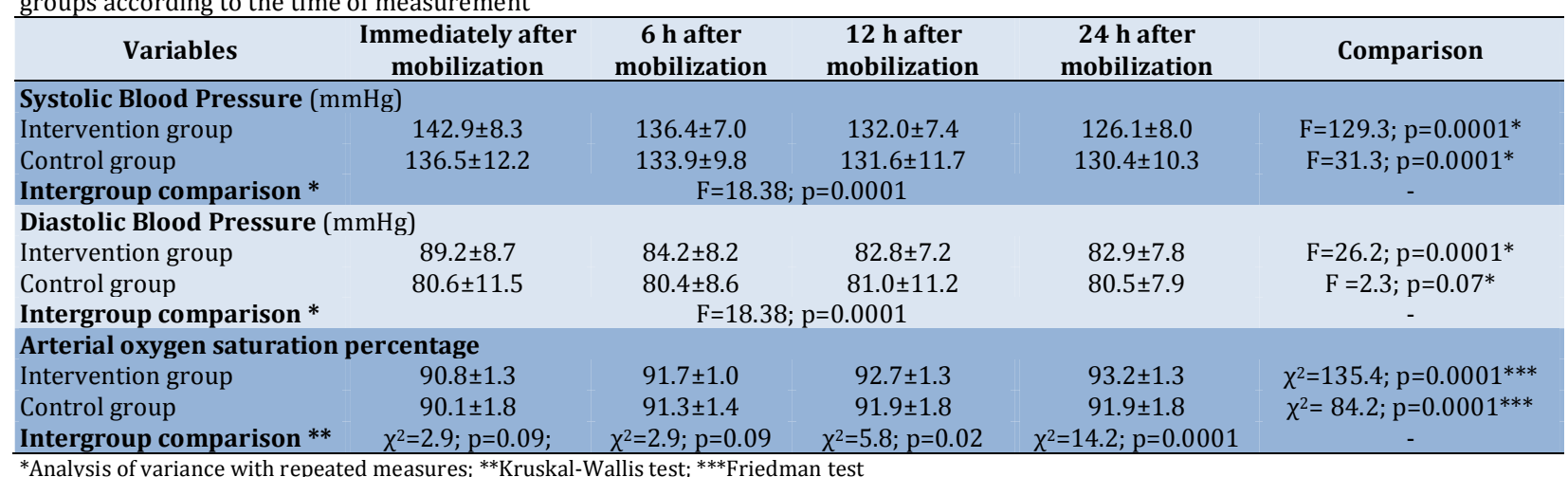

*Analysis of variance with repeated measures; ${ }^{* *}$ Kruskal-Wallis test; $* * *$ Friedman test

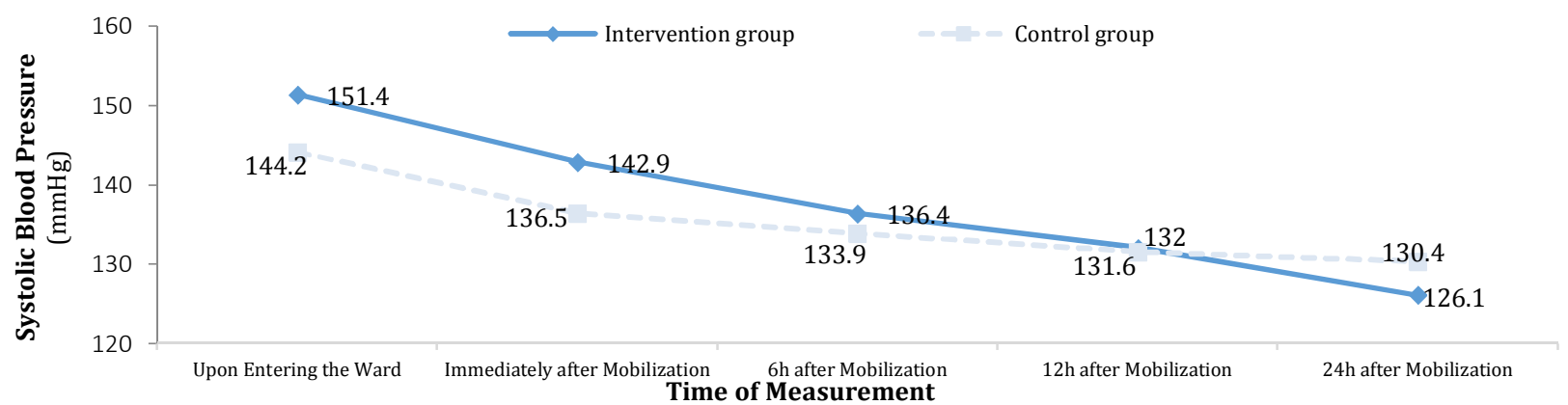

Diagram 1) Changes in mean systolic blood pressure of patients according to time of measurement

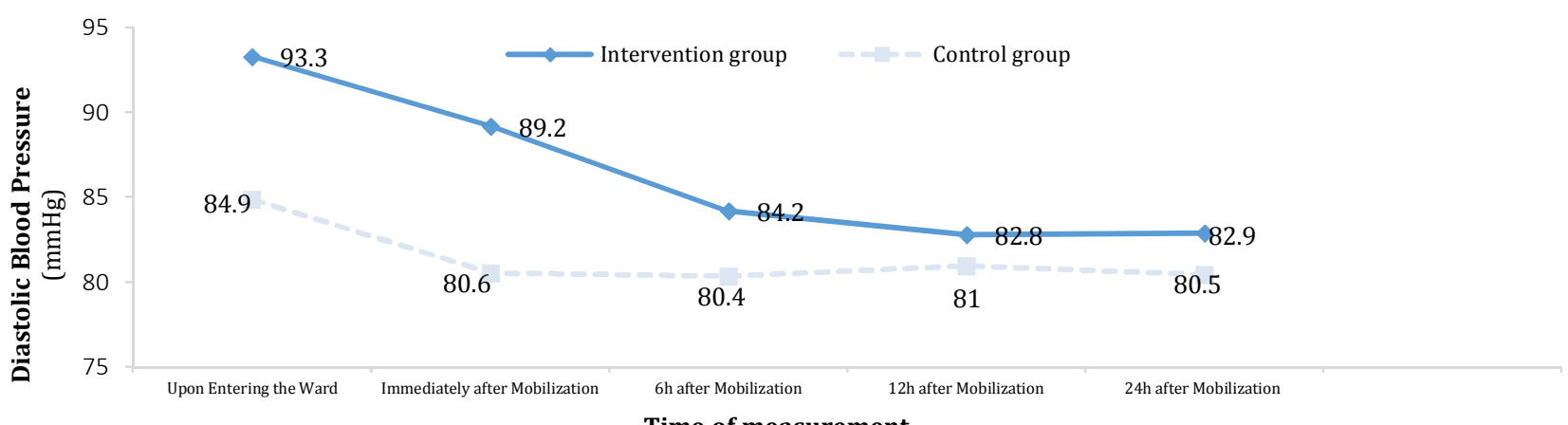

Time of measurement

Diagram 2) Changes in mean diastolic blood pressure of patients according to time of measurement 


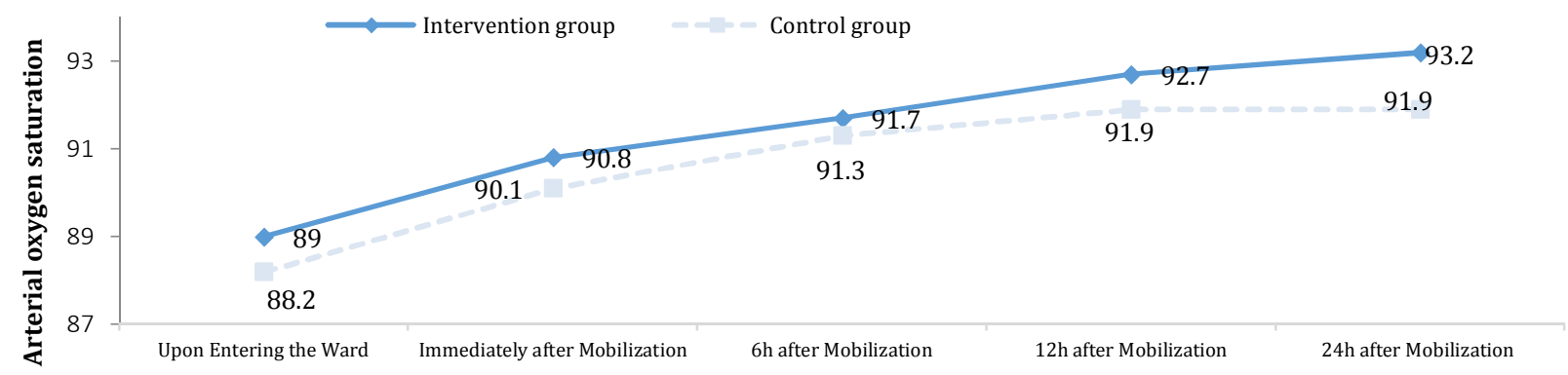

Time of Measurement

Diagram 3) Changes in mean arterial oxygen saturation of patients according to time of measurement

\section{Discussion}

Results of the study indicating a decrease in systolic and diastolic blood pressure and their descending slope in the intervention group compared to the control group and also its significant difference, as since after laparoscopic surgery, intra-abdominal pressure increases blood pressure, vascular resistance, and arterial hypertension [31], showed a faster recovery of systolic and diastolic blood pressure to normal level in the intervention group indicating favorable outcome of the intervention. However, the nonsignificant of intra-group comparisons of diastolic blood pressure at different measurement times in the control group indicates the effectiveness of intervention and the necessity of changes in routine procedure.

Arterial oxygen saturation decreases in patients undergoing laparoscopic surgery [15, 24], due to filling of the peritoneal cavity by carbon dioxide gas during surgery. As the diaphragm rises, pressure inside the chest also increases, which also affects lung capacity [31]. Increasing the rate and ascending slope in arterial oxygen saturation in the intervention group compared to the control group showed the effectiveness of gradual mobilization with activity in bed than the control group. The lack of significant difference between the groups in an increase in arterial oxygen saturation immediately after mobilization and $6 \mathrm{~h}$ later in the intervention and control groups indicated distracted ventilation associated with the filling of peritoneal cavity with the gas; however, the gradual discharge of this gas caused a statistically significant difference between the intervention and control groups at 12 and $24 \mathrm{~h}$ after the first mobilization.

Regarding the statistically significant changes in systolic blood pressure, diastolic blood pressure and arterial oxygen saturation, the findings of this study were comparable with the results of other relevant studies as follows:

Regarding systolic and diastolic blood pressure, the results of a study by Younis and Ahmed were consistent with the results of the present study; so that the systolic and diastolic blood pressure decreased significantly after motions in bed as a research intervention [32]. However, in contrast to this study, some studies [33-35] are not consistent with the findings of the present study indicating that systolic and diastolic pressure changes are caused by mobilization. Accordingly, there was no statistical difference between the intervention and control groups in the hemodynamic parameters immediately, 6, 12, and $24 \mathrm{~h}$ after the intervention. To explain this difference, it can be noted that in this study the motions involved the whole body, but in other (inconsistent) studies, the lower extremities were involved.

Comparison of the results of this study with other studies on arterial oxygen saturation showed that in the present study, arterial oxygen saturation in the intervention group increased faster than the control group and reached a balance, which is not in line with the results of the Younis and Ahmed study, which reported a decrease in arterial oxygen saturation. Difference in the results is probably because the only involvement of lower extremities without a gradual mobilization [32].

Also the results of this study were not in line with those of Şenduran et al., as there was no significant difference in heart rate, systolic and diastolic blood pressure, mean arterial pressure, and peripheral oxygen saturation at three time points before mobilization, immediately after mobilization, and 5 minutes after returning to bed. However, in the present study, the difference in hemodynamic factors was significant at the mentioned time points. The contrast in the results may have been due to a variety of surgeries, including upper abdominal surgery, liver transplantation, lower abdominal surgery, as well as upper and lower abdominal surgery, orthopedic surgery, and esophageal resection surgery ${ }^{[36]}$.

The study, conducted by Genc et al., supports the present study, which found that the movement of obese patients in intensive care units was associated with an increase in arterial oxygen saturation [37]. However the study by Rezaeikia et al. [29], which did not have a statistically significant difference in arterial oxygen saturation and control group, does not support this study. This may be due to inactivity 
in the lower extremities, however, in the recent study, movements in the bed was performed on the entire body.

One of the uncontrolled limitations was the use of specific medications or interventions that the patient possibly received within $24 \mathrm{~h}$ of the intervention affecting hemodynamic parameters.

Considering the effect of gradual mobilization with activity in bed on hemodynamic parameters in patients undergoing sleeve gastrectomy, it is recommended that the effect of gradual mobilization with activity in bed on other surgeries that affect hemodynamic parameters be investigated.

\section{Conclusion}

Gradual mobilization with activity in bed is effective in faster recovery of systolic blood pressure, diastolic blood pressure, and arterial oxygen saturation as hemodynamic parameters to normal range in patients undergoing sleeve gastrectomy.

Acknowledgments: This study was extracted from a Master's thesis in Nursing and Midwifery in the Yasuj University of Medical Sciences and funded by this University. The authors are grateful to the staff of Yasuj University of Medical Sciences, Department of Nursing and Midwifery, nurses of the Ghadir Mother and Child Hospital affiliated to the Shiraz University of Medical Sciences, the participants, including patients undergoing surgery, and all those who contributed to this study.

Ethical Permission: This study was approved by the Research Ethics Committee (IR.YUMS.REC.1395.30) and registered at the Iranian Registry of Clinical Trials (IRCT2016051527904N1).

Conflict of Interests: There is no conflict of interests.

Authors' Contribution: Kouhpeima MR. (First author), Introduction author/ Assistant (30\%); Sadat SJ. (Second author), Introduction author/ Original researcher/ Discussion author (40\%); Zoladl M. (Third author), Introduction author/ Assistant/ Statistical analyst/ Discussion author (25\%); Afrasiabifar A. (Fourth author), Introduction author/ Methodologist/ Assistant (5\%)

Funding: This study was funded by the Yasuj University of Medical Sciences.

\section{References}

1- Seki Y, Kasama K. Current status of laparoscopic bariatric surgery. Surg Technol Int. 2010;20:139-44.

2- Ghorbani M, Talebi H, Tavakoli M, Abdolmajidi F. The effect of central obesity on the auditory threshold of 25 to 40-years-old men and women. J Res Rehabil Sci. 2017;13(4):233-8. [Persian]

3- Chang S-H, Stoll CR, Colditz GA. Cost-effectiveness of bariatric surgery: should it be universally available? Maturitas. 2011;69(3):230-8.

4- Sarvghadi F, Rambod M, Hosseinpanah F, Hedayati M, Tohidi M, Azizi F. Prevalence of obesity in subjects aged 50 years and over in Tehran. Iran J Endocrinol Metab. 2007;9(1):99-104. [Persian]

5- Mehrabian F, Omidi S, Mahdavi Roshan M, Mirzaei M. Correlation between nutritional behavior, obesity, and overweight in female elementary school students in Anzali. J Health. 2018;9(3):291-301. [Persian]
6- Ogden J. The psychology of eating: from healthy to disordered behavior. 2nd Edition. Hoboken: Wiley; 2011.

7- McTigue KM, Harris R, Hemphill B, Lux L, Sutton S, Bunton AJ, et al. Screening and interventions for obesity in adults: summary of the evidence for the US Preventive Services Task Force. Ann Intern Med. 2003;139(11):93349.

8- Bessesen DH. Update on obesity. J Clin Endocrinol Metab. 2008;93(6):2027-34.

9- Piazza L, Ferrara F, Leanza S, Coco D, Sarvà S, Bellia A, et al. Laparoscopic mini-gastric bypass: short-term singleinstitute experience. Updates Surg. 2011;63(4):239-42.

10- Sheikhtaheri A, Beitollahi M, Pazouki A, Orooji A. Effective factors of mini gastric bypass surgery complications. Razi J Med Sci. 2018;25(168):48-58. [Persian]

11- Birkmeyer NJ, Dimick JB, Share D, Hawasli A, English WJ, Genaw J, et al. Hospital complication rates with bariatric surgery in Michigan. JAMA. 2010;304(4):435-42. 12- Schernthaner G, Morton JM. Bariatric surgery in patients with morbid obesity and type 2 diabetes. Diabetes Care. 2008;31(Suppl 2):S297-302.

13- Biörserud C. Excess skin after bariatric surgerypatients' perspective and objective measurements [Dissertation]. Gothenburg, Sweden: University of Gothenburg; 2015.

14- Gagner M, Deitel M, Kalberer TL, Erickson AL, Crosby RD. The second international consensus summit for sleeve gastrectomy, March 19-21, 2009. Surg Obes Relat Dis. 2009;5(4):476-85.

15- Karamanakos SN, Vagenas K, Kalfarentzos F, Alexandrides TK. Weight loss, appetite suppression, and changes in fasting and postprandial ghrelin and peptideYY levels after Roux-en-Y gastric bypass and sleeve gastrectomy: a prospective, double blind study. Ann Surg. 2008;247(3):401-7.

16- Ramos AC, Bastos EL, Ramos MG, Bertin NT, Galvao TD, de Lucena RT, et al. Medium-term follow-up results with laparoscopic sleeve gastrectomy. Arq Bras Cir Dig. 2015;28 Suppl 1:61-4. [English, Portuguese]

17- ASMBS Clinical Issues Committee. Updated position statement on sleeve gastrectomy as a bariatric procedure. Surg Obes Relat Dis. 2012;8(3):e21-6.

18- Moir J, Mc Callum I, Seymour K. The effect of bariatric surgery on bone health. Br J Med Med Res. 2015;8(2):11022.

19- Rubinshtein R, Ciubotaru M, Elad H, Bitterman H. Severe orthostatic hypotension following weight reduction surgery. Arch Intern Med. 2001;161(17):21457.

20- Billakanty SR, Kligman MD, Kanjwal YM, Kosinski DJ, Maly GT, Karabin B, et al. New-onset orthostatic intolerance following bariatric surgery. Pacing Clin Electrophysiol. 2008;31(7):884-8.

21- Abu-Jaish W, Patel R, Mohammad Jafferj M. Orthostatic intolerance following laparoscopic sleeve gastrectomy for morbid obesity. Bariatric Times. 2014;11(7):8-10.

22- Jacob G, Costa F, Shannon JR, Robertson RM, Wathen M, Stein M, et al. The neuropathic postural tachycardia syndrome. N Engl J Med. 2000;343(14):1008-14.

23- Shibao C, Lipsitz LA, Biaggioni I, American Society of Hypertension Writing Group. Evaluation and treatment of orthostatic hypotension. J Am Soc Hypertens. 2013;7(4):317-24.

24- Omalu BI, Luckasevic T, Shakir AM, Rozin L, Wecht CH, Kuller LH. Postbariatric surgery deaths, which fall under 
the jurisdiction of the coroner. Am J Forensic Med Pathol. 2004;25(3):237-42.

25- Koohpyma MR, Sadat SJ, Afrasiabifar A, Zoladl M. Effect of early mobilization on hemodynamic parameters of patients undergoing sleeve gastrectomy; a randomized clinical trial. J Clin Care Skills. 2019;1(2):55-61.

26- Parry SM, Puthucheary ZA. The impact of extended bed rest on the musculoskeletal system in the critical care environment. Extrem Physiol Med. 2015;4(1):16.

27- Mechanick JI, Youdim A, Jones DB, Garvey WT, Hurley DL, McMahon MM, et al. Clinical practice guidelines for the perioperative nutritional, metabolic, and nonsurgical support of the bariatric surgery patient-2013 update: cosponsored by American Association of Clinical Endocrinologists, the Obesity Society, and American Society for Metabolic \& Bariatric Surgery. Obesity. 2013;21(Suppl 1):S1-27.

28- Browning L, Denehy L, Scholes RL. The quantity of early upright mobilisation performed following upper abdominal surgery is low: an observational study. Aust J Physiother. 2007;53(1):47-52.

29- Rezaeikia R Najafi Doulatabad S, Afrasiabifar A, Zoladl M. Effect of passive movements of lower extremity on hemodynamic parameters of the patients under ventilator. J Clin Care Skills. 2019;1(1):37-42.

30- Barth MM, Jenson CE. Postoperative nursing care of gastric bypass patients. Am J Crit Care. 2006;15(4):378-87.
31- O'Malley C, Cunningham AJ. Physiologic changes during laparoscopy. Anesthesiol Clin North Am. 2001;19(1):1-19.

32- Younis GA, Ahmed SES. Effectiveness of passive range of motion exercise on hemodynamic parameters and behavioral pain intensity among adult mechanically ventilated patients. IOSR J Nurs Health Sci. 2015;4(6):4759.

33- Thelandersson A, Volkmann R, Cider Å. Blood flow velocity and vascular resistance during passive leg exercise in the critically ill patient. Clin Physiol Funct Imaging. 2012;32(5):338-42.

34- Fukuda S, Shimada K, Kawasaki T, Kono Y, Jissho S, Taguchi H, et al. "Passive exercise" using whole body periodic acceleration: effects on coronary microcirculation. Am Heart J. 2010;159(4):620-6.

35- Achugbue FS. The effects of therapeutic passive movement on cardiovascular response in stroke patients. Res J Med Sci. 2009;3(1):12-5.

36- Şenduran M, Arzu G, Mert A, Günerli A. Effects of mobilization on hemodynamic and respiratory responses in critically ill patients. Fizyoter Rehabil J. 2012;23(1):3-9.

37- Genc A, Ozyurek S, Koca U, Gunerli A. Respiratory and hemodynamic responses to mobilization of critically ill obese patients. Cardiopulm Phys Ther J. 2012;23(1):148. 